\title{
Duals of Hardy spaces on homogeneous groups
}

\author{
Marcin Bownik*1 and Gerald B. Folland**2 \\ ${ }^{1}$ Department of Mathematics, University of Oregon, Eugene, OR 97403-1222, USA \\ ${ }^{2}$ Department of Mathematics, University of Washington, Seattle, WA 98195-4350, USA
}

Received 13 January 2005, accepted 24 July 2005

Published online 9 July 2007

Key words Hardy space, Campanato space, homogeneous group, duality

MSC (2000) Primary: 42B30; Secondary: 43A80, 46E35

Hardy spaces on homogeneous groups were introduced and studied by Folland and Stein [3]. The purpose of this note is to show that duals of Hardy spaces $H^{p}, 0<p \leq 1$, on homogeneous groups can be identified with Morrey-Campanato spaces. This closes a gap in the original proof of this fact in [3].

(c) 2007 WILEY-VCH Verlag GmbH \& Co. KGaA, Weinheim

\section{Introduction}

We begin by reviewing some definitions. Let $G$ be a homogeneous group, i.e., $G$ is a connected and simply connected nilpotent Lie group which is endowed with a family of dilations $\left\{\delta_{r}\right\}_{r>0}$. We recall that a family of dilations on the Lie algebra $\mathfrak{g}$ of $G$ is a one parameter family of automorphisms of $\mathfrak{g}$ of the form $\{\exp (A \log r)$ : $r>0\}$, where $A$ is diagonalizable linear operator on $\mathfrak{g}$ with positive eigenvalues $1=d_{1} \leq d_{2} \leq \cdots \leq d_{n}$, $n=\operatorname{dim}(G)$. Then the exponential map from $\mathfrak{g}$ to $G$ defines the corresponding family of dilations $\left\{\delta_{r}\right\}_{r>0}$ on $G$. We will often use the abbreviated notation $\delta_{r} x=r x$ for $x \in G$ and $r>0$.

We fix a homogeneous norm on $G$, i.e., a continuous map $|\cdot|: G \rightarrow[0, \infty)$ that is $C^{\infty}$ on $G \backslash\{0\}$ and satisfies

$$
\begin{array}{ll}
\left|x^{-1}\right|=|x| & \text { for all } \quad x \in G, \\
\left|\delta_{r} x\right|=r|x| & \text { for all } \quad x \in G, r>0, \\
|x|=0 \Longleftrightarrow x=0 . &
\end{array}
$$

The ball $B(r, x)$ of radius $r>0$ and center $x \in G$ is defined as

$$
B(r, x)=\left\{y \in G:\left|x^{-1} y\right|<r\right\},
$$

and we denote by $\gamma$ be the minimal constant such that

$$
|x y| \leq \gamma(|x|+|y|) \text { for all } x, y \in G .
$$

If $\psi$ is a function on $G$ and $t>0$, we define its dilate $D_{t} \psi$ as

$$
D_{t} \psi(x)=t^{-Q} \psi\left(\delta_{1 / t} x\right)=t^{-Q} \psi(x / t),
$$

where

$$
Q=d_{1}+\cdots+d_{n}
$$

is the homogeneous dimension of $G$. The dilate $D_{t} \psi$ is also denoted by $\psi_{t}$. The (left) translate of $\psi$ by $x_{0} \in G$ is defined as

$$
\tau_{x_{0}} \psi(x)=\psi\left(\left(x_{0}\right)^{-1} x\right)
$$

* e-mail: mbownik@uoregon.edu, Phone: +1 541346 5622, Fax: +1 5413460987

** Corresponding author: e-mail: folland@math.washington.edu, Phone: +1 206543 7083, Fax: +1 2065430397 
Given a multiindex $I=\left(i_{1}, \ldots, i_{n}\right) \in \mathbb{N}^{n}$, we set

$$
|I|=i_{1}+\cdots+i_{n}, \quad d(I)=d_{1} i_{1}+\cdots+d_{n} i_{n} .
$$

Let $\Delta$ be the additive semi-group of $\mathbb{R}$ generated by $0, d_{1}, d_{2}, \ldots, d_{n}$. That is, $\Delta=\left\{d(I): I \in \mathbb{N}^{N}\right\}$. Let $\eta_{1}, \ldots, \eta_{n}$ be a basis for the linear polynomials on $G$ such that $\eta_{i}$ is homogeneous of degree $d_{i}$. Then every polynomial $P$ can be written uniquely as

$$
P=\sum_{I} a_{I} \eta^{I}, \quad \eta^{I}=\eta_{1}^{i_{1}} \ldots \eta_{n}^{i_{n}}, \quad a_{I} \in \mathbb{C} .
$$

The homogeneous degree of $P=\sum_{I} a_{I} \eta^{I}$ is defined as

$$
\operatorname{deg}(P)=\max \left\{d(I): a_{I} \neq 0\right\} .
$$

Given $s \in \Delta$, we denote the space of polynomials of homogeneous degree $\leq s$ by

$$
\mathcal{P}_{s}=\{P \in \mathcal{P}: \operatorname{deg}(P) \leq s\}
$$

We recall that $\mathcal{P}_{s}$ is invariant under left and right translations; see [3, Proposition 1.25].

Suppose that $0<p \leq 1,1 \leq q \leq \infty$ and $s \in \Delta$. We say that a triplet $(p, q, s)$ is admissible if $p<q$ and

$$
s \geq \max \left\{s^{\prime} \in \Delta: s^{\prime} \leq Q(1 / p-1)\right\} .
$$

We say that a function $a$ is a $(p, q, s)$-atom, where $(p, q, s)$ is admissible, if

$$
\begin{aligned}
& \text { supp } a \subset B\left(x_{0}, r\right) \text { for some } x_{0} \in G, \quad r>0, \\
& \|a\|_{q} \leq\left|B\left(x_{0}, r\right)\right|^{1 / q-1 / p} \\
& \int_{G} a(x) P(x) d x=0 \text { for all } P \in \mathcal{P}_{s} .
\end{aligned}
$$

The atomic Hardy space $H_{q, s}^{p}$ is the set of all tempered distributions $f$ such that $f=\sum \lambda_{i} a_{i}$ (convergence in $\mathcal{S}^{\prime}$ ) such that the $a_{i}$ are $(p, q, s)$-atoms, $\lambda_{i} \geq 0$, and $\sum \lambda_{i}^{p}<\infty$. $H_{q, s}^{p}$ is actually independent of $q$ and $s$ ([3, Theorem 3.30]) and so may be denoted simply by $H^{p}$.

Let $\mathcal{B}$ denote the collection of all open balls in $G$. If $l \geq 0,1 \leq q \leq \infty$, and $s \in \Delta$, we define the Campanato space $C_{q, s}^{l}$ to be the space of all locally $L^{q}$ functions $u$ on $G$ so that

$$
\begin{aligned}
\|u\|_{C_{q, s}^{l}} & :=\sup _{B \in \mathcal{B}} \inf _{P \in \mathcal{P}_{s}}|B|^{-l}\left(\frac{1}{|B|} \int_{B}|u(x)-P(x)|^{q} d x\right)^{1 / q}<\infty \quad(q<\infty), \\
\|u\|_{C_{\infty, s}^{l}} & :=\sup _{B \in \mathcal{B}} \inf _{P \in \mathcal{P}_{s}}|B|^{-l} \operatorname{ess}_{\sup _{x \in B}|u(x)-P(x)|<\infty} \quad(q=\infty) .
\end{aligned}
$$

We identify two elements of $C_{q, s}^{l}$ if they are equal almost everywhere. (Note: The space called $C_{q, s}^{l}$ here is called $C_{q, s}^{Q l}$ in [3].)

\section{Duals of Hardy spaces}

The main goal of this note is to prove that the dual of the Hardy space $H_{q, s}^{p}$ is isomorphic to the Campanato space $C_{q^{\prime}, s}^{1 / p-1} / \mathcal{P}_{s}$, where $(p, q, s)$ is an admissible triplet and $1 / q+1 / q^{\prime}=1$. This result in the setting of Hardy spaces on homogeneous groups was obtained by Folland and Stein [3, Chapter 5]. However, careful examination of the arguments in [3] reveals a gap in the first part of the proof of [3, Theorem 5.3]. The trouble is that uniform boundedness of a functional on atoms does not guarantee that the functional is bounded on $H^{p}$; see [2]. Hence, the operator norm of a functional $L$ on $H^{p}$ is given by the supremeum of $|L a|$ over all atoms $a$, as asserted in [3, Lemma 5.1], only when the functional is known a priori to be continuous. To remedy this situation we will apply a rather subtle approximation argument inspired by [4, Chapter III.5], see also [1, Section 8]. 
We will need some simple observations about Campanato spaces. First, note that for any $t>0$, the substitution $s=r / t$ gives

$$
\begin{aligned}
\left\|u_{t}\right\|_{C_{q^{\prime}, s}^{l}} & =\sup _{x_{0} \in G, r>0} \inf _{P \in \mathcal{P}_{s}}\left|B\left(x_{0}, r\right)\right|^{-l}\left(\frac{1}{\left|B\left(x_{0}, r\right)\right|} \int_{B\left(x_{0}, r\right)}\left|t^{-Q} u(x / t)-P(x)\right|^{q^{\prime}} d x\right)^{1 / q^{\prime}} \\
& =\sup _{x_{0} \in G, s>0} \inf _{P \in \mathcal{P}_{s}}\left(t^{Q}\left|B\left(x_{0}, s\right)\right|\right)^{-l}\left(\frac{1}{\left|B\left(x_{0}, s\right)\right|} \int_{B\left(x_{0}, s\right)} t^{-Q q^{\prime}}\left|u(x)-t^{Q} P(t x)\right|^{q^{\prime}} d x\right)^{1 / q^{\prime}} \\
& =t^{-Q(l+1)}\|u\|_{C_{q^{\prime}, s}^{l}} .
\end{aligned}
$$

Next, for any $B \in \mathcal{B}$, let $\pi_{B}: L^{1}(B) \rightarrow \mathcal{P}_{s}$ be the natural projection defined by

$$
\int_{B}\left(\pi_{B} f(x)\right) Q(x) d x=\int_{B} f(x) Q(x) d x \quad \text { for all } f \in L^{1}(B), \quad Q \in \mathcal{P}_{s} .
$$

We claim that there is a constant $C=C_{s}$, independent of $f$ and $B$, such that

$$
\sup _{x \in B}\left|\pi_{B} f(x)\right| \leq C \frac{1}{|B|} \int_{B}|f(x)| d x .
$$

Indeed, for the fixed ball $B_{0}=B(0,1)$, let $\left\{Q_{I}: d(I) \leq s\right\}$ be an orthonormal basis of $\mathcal{P}_{s}$ with respect to the $L^{2}\left(B_{0}\right)$ norm. Then

$$
\pi_{B_{0}} f=\sum_{d(I) \leq s}\left(\int_{B_{0}} f(x) \overline{Q_{I}(x)} d x\right) Q_{I},
$$

so the estimate (2.2) holds for $B=B_{0}$ with $C=\left|B_{0}\right| \sum_{I}\left(\sup _{x \in B_{0}}\left|Q_{I}(x)\right|\right)^{2}$. Since $\pi_{B\left(x_{0}, r\right)} f=\left(\tau_{x_{0}} \circ D_{r} \circ\right.$ $\left.\pi_{B_{0}} \circ D_{1 / r} \circ \tau_{\left(x_{0}\right)^{-1}}\right) f,(2.2)$ then follows for arbitrary $B=B\left(x_{0}, r\right) \in \mathcal{B}$.

Next, we claim that we can define an equivalent norm on $C_{q^{\prime}, s}^{l}$ by setting

$$
\begin{aligned}
\||u|\|_{C_{q^{\prime}, s}^{l}} & =\sup _{B \in \mathcal{B}}|B|^{-l}\left(\frac{1}{|B|} \int_{B}\left|u(x)-\pi_{B} u(x)\right|^{q^{\prime}} d x\right)^{1 / q^{\prime}} & & \left(1 \leq q^{\prime}<\infty\right), \\
\|\| u \|_{C_{\infty, s}^{l}}^{l} & =\sup _{B \in \mathcal{B}}|B|^{-l} \operatorname{ess}_{\sup _{x \in B}\left|u(x)-\pi_{B} u(x)\right|} & & \left(q^{\prime}=\infty\right) .
\end{aligned}
$$

Indeed, for any $B \in \mathcal{B}$ and $P \in \mathcal{P}_{s}$, by the fact that $P=\pi_{B} P$ and (2.2) we have

$$
\begin{aligned}
& \left(\frac{1}{|B|} \int_{B}\left|u(x)-\pi_{B} u(x)\right|^{q^{\prime}} d x\right)^{1 / q^{\prime}} \\
& \quad \leq\left(\frac{1}{|B|} \int_{B}|u(x)-P(x)|^{q^{\prime}} d x\right)^{1 / q^{\prime}}+\left(\frac{1}{|B|} \int_{B}\left|\pi_{B}(P-u)(x)\right|^{q^{\prime}} d x\right)^{1 / q^{\prime}} \\
& \quad \leq\left(\frac{1}{|B|} \int_{B}|u(x)-P(x)|^{q^{\prime}} d x\right)^{1 / q^{\prime}}+C \frac{1}{|B|} \int_{B}|u(x)-P(x)| d x \\
& \quad \leq(C+1)\left(\frac{1}{|B|} \int_{B}|u(x)-P(x)|^{q^{\prime}} d x\right)^{1 / q^{\prime}} .
\end{aligned}
$$

Therefore,

$$
\|u\|_{C_{q^{\prime}, s}^{l}} \leq\|\| u\|\|_{C_{q^{\prime}, s}^{l}} \leq(C+1)\|u\|_{C_{q^{\prime}, s}^{l}} \text { for all } u \in C_{q^{\prime}, s}^{l} .
$$

The key ingredients in the proof of the duality theorem are some approximation results for Campanato spaces. To begin with, define the space

$$
\Theta_{s}^{q}=\left\{f \in L^{q}(G): \operatorname{supp} f \text { is compact and } \int_{G} f(x) P(x) d x=0 \text { for } P \in \mathcal{P}_{s}\right\} .
$$


Lemma 2.1 Suppose $u \in C_{q^{\prime}, s}^{l}$, where $l \geq 0,1 \leq q^{\prime} \leq \infty$, and $s=0,1, \ldots$ Fix a nonnegative function $\varphi \in C^{\infty}$ with compact support and $\int \varphi=1$, and let $\varphi_{r}(x)=r^{-Q} \varphi(x / r)$. Then

$$
\int_{G} f(x)\left(u * \varphi_{r}\right)(x) d x \longrightarrow \int_{G} f(x) u(x) d x \quad \text { as } \quad r \longrightarrow 0 \text { for all } f \in \Theta_{s}^{q},
$$

and

$$
\left\|u * \varphi_{r}\right\|_{C_{q^{\prime}, s}^{l}} \leq\|\| u \|_{C_{q^{\prime}, s}^{l}} \text { for all } r>0 .
$$

Proof. If $q^{\prime}<\infty,(2.7)$ holds since $u * \varphi_{r} \rightarrow u$ in $L_{\mathrm{loc}}^{q^{\prime}}(G)$ as $r \rightarrow 0$. If $q^{\prime}=\infty, u * \varphi_{r}$ is uniformly bounded on compact sets and converges a.e. to $u(x)$ by [3, Theorem 2.6], so (2.7) holds by the dominated convergence theorem. Next, given $B \in \mathcal{B}$ and $r>0$, define a function $P_{r}$ by

$$
P_{r}(x)=\int_{G} \pi_{y^{-1} B} u\left(y^{-1} x\right) \varphi_{r}(y) d y .
$$

Since we can write $\pi_{y^{-1} B} u\left(y^{-1} x\right)=\sum_{d(I) \leq s} c_{\alpha}(y) \eta^{I}\left(y^{-1} x\right)$ and the coefficients $c_{\alpha}(y)$ are continuous functions of $y, P_{r}$ is a polynomial of homogeneous degree $\leq s$. By the Minkowski inequality,

$$
\begin{aligned}
& \left(\frac{1}{|B|} \int_{B}\left|\left(u * \varphi_{r}\right)(x)-P_{r}(x)\right|^{q^{\prime}} d x\right)^{1 / q^{\prime}} \\
& \quad=\left(\frac{1}{|B|} \int_{B}\left|\int_{G}\left(u\left(y^{-1} x\right)-\pi_{y^{-1} B} u\left(y^{-1} x\right)\right) \varphi_{r}(y) d y\right|^{q^{\prime}} d x\right)^{1 / q^{\prime}} \\
& \quad \leq \int_{G}\left(\frac{1}{|B|} \int_{B}\left|u\left(y^{-1} x\right)-\pi_{y^{-1} B} u\left(y^{-1} x\right)\right|^{q^{\prime}} d x\right)^{1 / q^{\prime}}\left|\varphi_{r}(y)\right| d y \\
& \quad=\int_{G}\left(\frac{1}{\left|y^{-1} B\right|} \int_{y^{-1} B}\left|u(z)-\pi_{y^{-1} B} u(z)\right|^{q^{\prime}} d z\right)^{1 / q^{\prime}} \varphi_{r}(y) d y \\
& \leq\left.|||u|\right|_{C_{q^{\prime}, s}^{l}}|B|^{l} .
\end{aligned}
$$

This proves (2.8)

Lemma 2.2 Let $\psi \in C^{\infty}$ be such that $\operatorname{supp} \psi \subset B(0,1), 0 \leq \psi(x) \leq 1$, and $\psi(x)=1$ for $x \in B(0,1 / 2)$. There exist $C>0$ and $\tilde{s} \in \Delta$ with $\tilde{s} \geq s$ such that

$$
\left\|\left(u-\pi_{B_{0}} u\right) \psi\right\|_{C_{q^{\prime}, \tilde{s}}^{l}} \leq C\|u\|_{C_{q^{\prime}, s}^{l}} \text { for all } u \in C_{q^{\prime}, s}^{l},
$$

where $B_{0}=B(0, \gamma(2 \gamma+1))$.

Proof. Suppose $u \in C_{q^{\prime}, s}^{l}$ with \|\|$u \|_{C_{q^{\prime} s}^{l}} \leq 1$. For brevity, we only consider the case $q^{\prime}<\infty$; the case $q^{\prime}=\infty$ uses a similar argument. Let $U=u-\pi_{B_{0}} u$. Since $\operatorname{supp} \psi \subset B_{0}$,

$$
\int_{G}|U(x) \psi(x)|^{q^{\prime}} d x \leq \int_{B_{0}}|U(x)|^{q^{\prime}} d x \leq\left|B_{0}\right|^{l q^{\prime}+1}<\infty .
$$

Therefore, if $B=B\left(x_{0}, r\right) \in \mathcal{B}$ with $r \geq 1$, then

$$
|B|^{-l}\left(\frac{1}{|B|} \int_{B}|U(x) \psi(x)|^{q^{\prime}} d x\right)^{1 / q^{\prime}} \leq\left(\frac{\left|B_{0}\right|}{|B|}\right)^{l+1 / q^{\prime}} \leq C<\infty .
$$

Hence, to show (2.9) it is enough to estimate the integral of $U \psi$ over balls $B=B\left(x_{0}, r\right)$ with $0<r<1$. Moreover, we can assume that $B \cap B(0,1) \neq \varnothing$, since otherwise $U \psi=0$ on $B$. Consequently, we are limited to balls $B \subset B_{0}$. Let $P_{1}=\pi_{B} U$. By (2.2) and (2.10),

$$
\left(\frac{1}{|B|} \int_{B}\left|P_{1}(x)\right|^{q^{\prime}} d x\right)^{1 / q^{\prime}} \leq C\left(\frac{1}{|B|} \int_{B}|U(x)|^{q^{\prime}} d x\right)^{1 / q^{\prime}} \leq C_{1}|B|^{-1 / q^{\prime}} .
$$


Let $P_{2}(x)$ be the left Taylor polynomial of $\psi$ at $x_{0}$ of homogenous degree $s_{0}$ (i.e., the polynomial whose leftinvariant derivatives at the origin of homogeneous degree $\leq s_{0}$ agree with the corresponding derivatives of $f$ at $\left.x_{0}\right)$, where $s_{0} \in \Delta$ is chosen to satisfy $s_{0} \geq Q\left(l+1 / q^{\prime}\right)$. By the Taylor inequality ([3, Theorem 1.37] and the remark following it), the remainder satisfies

$$
\left|\psi(x)-P_{2}\left(x_{0}^{-1} x\right)\right| \leq C_{2}\left|x_{0}^{-1} x\right|^{s_{0}} \quad \text { for } \quad x \in B \subset B\left(x_{0}, 1\right),
$$

with $C_{2}$ independent of $x_{0}$. Finally, let $P(x)=P_{1}(x) P_{2}\left(x_{0}^{-1} x\right)$, which is a polynomial of homogeneous degree at most $\tilde{s}=s+s_{0}$. By (2.11),

$$
\begin{aligned}
& \left(\int_{B}|U(x) \psi(x)-P(x)|^{q^{\prime}} d x\right)^{1 / q^{\prime}} \\
& \quad \leq\left(\int_{B}\left|\left[U(x)-P_{1}(x)\right] \psi(x)\right|^{q^{\prime}} d x\right)^{1 / q^{\prime}}+\left(\int_{B}\left|P_{1}(x)\left[\psi(x)-P_{2}\left(x_{0}^{-1} x\right)\right]\right|^{q^{\prime}} d x\right)^{1 / q^{\prime}} \\
& \quad \leq\|\psi\|_{\infty}\left(\int_{B}\left|U(x)-P_{1}(x)\right|^{q^{\prime}} d x\right)^{1 / q^{\prime}}+\sup _{x \in B}\left|\psi(x)-P_{2}\left(x_{0}^{-1} x\right)\right|\left(\int_{B}\left|P_{1}(x)\right|^{q^{\prime}} d x\right)^{1 / q^{\prime}} \\
& \quad \leq|B|^{l+1 / q^{\prime}}+C_{1} C_{2} r^{s_{0}} .
\end{aligned}
$$

But $r^{s_{0}}=C_{3}|B|^{s_{0} / Q} \leq C_{3}|B|^{l+1 / q^{\prime}}$, so (2.9) is proved with $\tilde{s}=s+s_{0}$.

Lemma 2.3 Suppose $u \in C_{q^{\prime}, s}^{l}$, where $l \geq 0,1 \leq q^{\prime} \leq \infty$, and $s=0,1, \ldots$ There exist $\tilde{s} \geq s$, a constant $C>0$ independent of $u$, and a sequence of test functions $\left\{u_{k}\right\}_{k \in \mathbb{N}} \subset \mathcal{S}$ so that

$$
\begin{aligned}
& \left\|u_{k}\right\|_{C_{q^{\prime}, \tilde{s}}^{l}} \leq C\|u\|_{C_{q^{\prime}, s}^{l}} \quad \text { for all } \quad k \in \mathbb{N}, \\
& \lim _{k \rightarrow \infty} \int_{G} f(x) u_{k}(x) d x=\int_{G} f(x) u(x) d x \quad \text { for all } \quad f \in \Theta_{s}^{q}, \quad 1 / q+1 / q^{\prime}=1 .
\end{aligned}
$$

Proof. First suppose $u \in C_{q^{\prime}, s}^{l} \cap C^{\infty}$. Let $\tilde{u}_{k}=D_{2^{-k}} u$ and $u_{k}=D_{2^{k}}\left(\left(\tilde{u}_{k}-\pi_{B_{0}} \tilde{u}_{k}\right) \psi\right)$, where $B_{0}$ is as in Lemma 2.2. By (2.1) and (2.9),

$$
\left\|\left(\tilde{u}_{k}-\pi_{B_{0}} \tilde{u}_{k}\right) \psi\right\|_{C_{q^{\prime}, \tilde{s}}^{l}} \leq C\left\|\tilde{u}_{k}\right\|_{C_{q^{\prime}, s}^{l}}=C 2^{k Q(l+1)}\|u\|_{C_{q^{\prime}, s}^{l}} .
$$

Therefore (2.12) holds, since

$$
\left\|u_{k}\right\|_{C_{q^{\prime}, \tilde{s}}^{l}}=2^{-k Q(l+1)}\left\|\left(\tilde{u}_{k}-\pi_{B_{0}} \tilde{u}_{k}\right) \psi\right\|_{C_{q^{\prime}, \tilde{s}}^{l}} \leq C\|u\|_{C_{q^{\prime}, s}^{l}} .
$$

Moreover,

$$
u_{k}(x)=u(x)-\left(D_{2^{k}} \circ \pi_{B_{0}} \circ D_{2^{-k}}\right) u(x)=u(x)-\pi_{B\left(0,2^{k} \gamma(2 \gamma+1)\right)} u(x) \quad \text { for } \quad x \in B\left(0,2^{k-1}\right) .
$$

Thus (2.13) also holds.

To end the proof we remove the assumption that $u \in C^{\infty}$. Given $u \in C_{q^{\prime}, s}^{l}$, define the sequence $\left\{u_{k}\right\}_{k \in \mathbb{N}} \subset \mathcal{S}$ by $u_{k}=D_{2^{k}}\left(\left(\tilde{u}_{k}-\pi_{B_{0}} \tilde{u}_{k}\right) \psi\right)$, where $\tilde{u}_{k}=D_{2^{-k}}\left(u * \varphi_{k}\right)$ with $\varphi_{k}$ as in Lemma 2.1. Combining (2.8) and (2.14) yields (2.12), whereas (2.7) and (2.15) yield (2.13), completing the proof of of Lemma 2.3.

Lemma 2.4 Suppose that $(p, q, s)$ is admissible and $f \in \Theta_{s}^{q}$, where $\Theta_{s}^{q}$ is given by (2.6). Suppose $u \in C_{q^{\prime}, s}^{l}$, $1 / q+1 / q^{\prime}=1, l=1 / p-1$. There exists $\tilde{s} \geq s$ such that if $f$ is decomposed into $f=\sum_{i=1}^{\infty} \lambda_{i} a_{i}$, where $\sum_{i=1}^{\infty}\left|\lambda_{i}\right|^{p}<\infty$ and the $a_{i}$ 's are $(p, q, \tilde{s})$-atoms, then

$$
\int f u=\sum_{i=1}^{\infty} \lambda_{i} \int a_{i} u .
$$


Proof. Let $a$ be a $(p, q, s)$-atom supported on a ball $B \in \mathcal{B}$ and $u \in C_{q^{\prime}, s}^{l}$. Since $\int u a=\int(u-P) a$ for all $P \in \mathcal{P}_{s}$ then by the standard calculation we have

$$
\begin{aligned}
\left|\int u a\right| & =\inf _{P \in \mathcal{P}_{s}}\left|\int(u-P) a\right| \\
& \leq\left(\int_{B}|a|^{q}\right)^{1 / q}\left(\inf _{P \in \mathcal{P}_{s}} \int|u-P|^{q^{\prime}}\right)^{1 / q^{\prime}} \\
& \leq|B|^{1 / q-1 / p}|B|^{l+1 / q^{\prime}}|B|^{-l}\left(\frac{1}{|B|} \inf _{P \in \mathcal{P}_{s}} \int|u-P|^{q^{\prime}}\right)^{1 / q^{\prime}} \\
& \leq\|u\|_{C_{q^{\prime}, s}^{l} .}
\end{aligned}
$$

Next, suppose that $f \in \Theta_{s}^{q}$ is decomposed into $f=\sum_{i=1}^{\infty} \lambda_{i} a_{i}$, where $\sum_{i=1}^{\infty}\left|\lambda_{i}\right|^{p}<\infty$ and the $a_{i}$ 's are $(p, q, \tilde{s})$-atoms, where $\tilde{s} \geq s$ is the same as in Lemma 2.3. Suppose also that $u \in C_{q^{\prime}, s}^{l}, 1 / q+1 / q^{\prime}=1$, $l=1 / p-1$. Let $\left\{u_{k}\right\}_{k \in \mathbb{N}} \subset \mathcal{S}$ be the sequence guaranteed by Lemma 2.3. For every $k \in \mathbb{N}$ we have

$$
\int f u_{k}=\sum_{i=1}^{\infty} \lambda_{i} \int a_{i} u_{k}
$$

since convergence in $H^{p}$ implies convergence in $\mathcal{S}^{\prime}$ by [3, Proposition 2.15]. By (2.13)

$$
\lim _{k \rightarrow \infty} \int_{G} a_{i}(x) u_{k}(x) d x=\int_{G} a_{i}(x) u(x) d x \quad \text { for all } \quad i \in \mathbb{N} .
$$

By (2.12) and (2.17) we have $\left|\int u_{k} a_{i}\right| \leq\left\|u_{k}\right\|_{C_{q^{\prime}, \tilde{s}}^{l}} \leq C\|u\|_{C_{q^{\prime}, s}^{l}}$. Since $\sum_{i=1}^{\infty}\left|\lambda_{i}\right| \leq\left(\sum_{i=1}^{\infty}\left|\lambda_{i}\right|^{p}\right)^{1 / p}<\infty$ we can take the limit as $k \rightarrow \infty$ in (2.18) by the dominated convergence theorem applied to counting measure on $\mathbb{N}$. This shows (2.16).

At last we are in a position to prove the duality theorem.

Theorem 2.5 Suppose $(p, q, s)$ is admissible. Then

$$
\left(H_{q, s}^{p}\right)^{*} \cong C_{q^{\prime}, s}^{l} / \mathcal{P}_{s}, \quad \text { where } 1 / q+1 / q^{\prime}=1, \quad l=1 / p-1
$$

More precisely, if $u \in C_{q^{\prime}, s}^{l}$ and $f$ is a finite linear combination of $(p, q, s)$-atoms, let $L_{u} f=\int u f$. Then $L_{u}$ extends continuously to $H_{q, s}^{p}$, and every $L \in\left(H_{q, s}^{p}\right)^{*}$ is of this form. Moreover,

$$
\|u\|_{C_{q^{\prime}, s}^{l}}=\left\|L_{u}\right\|_{\left(H_{q, s}^{p}\right)^{*}} \text { for all } u \in C_{q^{\prime}, s}^{l} .
$$

Proof. The fact that any bounded functional $L$ on $H_{q, s}^{p}$ must be of the form $L=L_{u}$ for some $u \in C_{q^{\prime}, s}^{l}$ was already shown in [3].

Conversely, suppose $u \in C_{q^{\prime}, s}^{l}$. Our goal is to demonstrate that the functional $L_{u} f=\int u f$ defined initially for $f \in \Theta_{s}^{q}$, where $\Theta_{s}^{q}$ is given by (2.6), extends to a bounded functional on $H_{q, s}^{p}$ and $\left\|L_{u}\right\|_{\left(H_{q, s}^{p}\right)^{*}} \leq\|u\|_{C_{q^{\prime}, s}^{l}}$. We emphasize again that boundedness of $L_{u}$ on atoms (2.17) alone does not guarantee boundedness on the entire space.

Suppose that $f \in \Theta_{s}^{q}$. By [3, Theorem 3.30] we can find an atomic decomposition of $f=\sum_{i=1}^{\infty} \lambda_{i} a_{i}$, where

$$
\left(\sum_{i=1}^{\infty}\left|\lambda_{i}\right|^{p}\right)^{1 / p} \leq 2\|f\|_{H_{q, \tilde{s}}^{p}} \leq C\|f\|_{H_{q, s}^{p}},
$$

and the $a_{i}$ 's are $(p, q, \tilde{s})$-atoms. By (2.17) and Lemma 2.4

$$
\left|L_{u} f\right| \leq \sum_{i=1}^{\infty}\left|\lambda_{i}\right|\left|L_{u} a_{i}\right| \leq\|u\|_{C_{q^{\prime}, s}^{l}}\left(\sum_{i=1}^{\infty}\left|\lambda_{i}\right|^{p}\right)^{1 / p} \leq C\|u\|_{C_{q^{\prime}, s}^{l}}\|f\|_{H_{q, s}^{p}} .
$$


Therefore, $L_{u}$ extends uniquely to a bounded functional on $H_{q, s}^{p}$. Next, we recall that the norm of a bounded functional on $H_{q, s}^{p}$ is always achieved on atoms; see [3, Lemma 5.1], which holds under the assumption of continuity. Therefore, (2.17) implies $\left\|L_{u}\right\|_{\left(H_{q, s}^{p}\right)^{*}} \leq\|u\|_{C_{q^{\prime}, s}^{l}}$, which finishes the proof of Theorem 2.5.

\section{References}

[1] M. Bownik, Anisotropic Hardy Spaces and Wavelets, Memoirs of the American Mathematical Society Vol. 164, No. 781 (Amer. Math. Soc., Providence, RI, 2003).

[2] M. Bownik, Boundedness of operators on Hardy spaces via atomic decompositions, Proc. Amer. Math Soc. 133, 35353542 (2005).

[3] G. B. Folland and E. M. Stein, Hardy Spaces on Homogeneous Groups (Princeton University Press, Princeton, NJ, 1982).

[4] J. García-Cuerva and J. L. Rubio de Francia, Weighted Norm Inequalities and Related Topics (North-Holland, Amsterdam, 1985). 